Research Paper

\title{
Trends in Treatment for Prostate Cancer in China: Preliminary Patterns of Care Study in a Single Institution
}

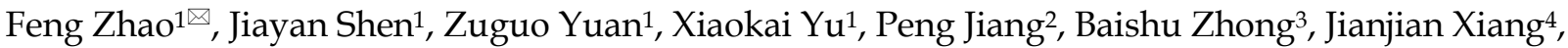 \\ Guoping Ren ${ }^{5}$, Liping $X^{2}{ }^{2}$, Senxiang Yan ${ }^{1 凶}$ \\ 1. Department of Radiation Oncology, the First Affiliated Hospital, College of Medicine, Zhejiang University, Hangzhou, Zhejiang 310003, P.R. China. \\ 2. Department of Urology, the First Affiliated Hospital, College of Medicine, Zhejiang University, Hangzhou, Zhejiang 310003, P.R. China. \\ 3. Department of Radiology, the First Affiliated Hospital, College of Medicine, Zhejiang University, Hangzhou 310003, Zhejiang Province, China. \\ 4. Department of Ultrasound, the First Affiliated Hospital, College of Medicine, Zhejiang University, Hangzhou, Zhejiang 310003, P.R. China. \\ 5. Department of Pathology, the First Affiliated Hospital, College of Medicine, Zhejiang University, Hangzhou, Zhejiang 310003, P.R. China.
}

$\square$ Corresponding author: Feng Zhao, Department of Radiation Oncology, the First Affiliated Hospital, College of Medicine, Zhejiang University, Hangzhou, Zhejiang 310003, P.R. China, Email: zju_zhaofeng@zju.edu.cn. Senxiang Yan, Department of Radiation Oncology, the First Affiliated Hospital, College of Medicine, Zhejiang University, Hangzhou, Zhejiang 310003, P.R. China, Email: yansenxiang@zju.edu.cn.

(c) Ivyspring International Publisher. This is an open access article distributed under the terms of the Creative Commons Attribution (CC BY-NC) license (https:// creativecommons.org/licenses/by-nc/4.0/). See http://ivyspring.com/terms for full terms and conditions.

Received: 2018.01.23; Accepted: 2018.03.13; Published: 2018.04.19

\begin{abstract}
Objectives: A Patterns of Care Study (PCS) was performed in the largest regional medical center in Zhejiang Province, China. The hospital information system (HIS) was used to evaluate patient characteristics and changes in initial treatment patterns for prostate cancer and to determine recent predominant trends in treatment plans for prostate cancer (PCa) in China.

Methods: Men who were newly diagnosed with localized or locally advanced PCa for 2010-2011 and 2016-2017 were identified in the HIS database. Patient characteristics and temporal trends in initial management were assessed, and differences between groups were evaluated for significance using Chi-square and Mann-Whitney $U$ tests.

Results: In total, 1792 patients met the study criteria, including 505 and 1287 patients in the 2010-2011 and 2016-2017 samples, respectively. The average age of patients diagnosed in the 2010-2011 PCS survey was 70 years, decreasing to 68 years when the 2016-2017 patients were included $(P<0.001)$. In the 2010-2011 sample, $50.69 \%$ of the patients had an initial prostate-specific antigen (PSA) level $\geq 20 \mathrm{ng} / \mathrm{ml}$. In contrast, the initial PSA level was $4-19.99 \mathrm{ng} / \mathrm{ml}$ for $66.67 \%$ of the patients in the $2016-2017$ sample $(P<0.001)$. Based on National Comprehensive Cancer Network (NCCN) criteria, the percentages of patients in low- and intermediate-risk groups increased from $33.06 \%$ to $54.78 \%$; conversely, the percentages in high-risk, very high-risk, and regional (N1) groups decreased to a certain extent $(\mathrm{P}<0.001)$. According to European Association of Urology (EAU) criteria, the percentages of patients in low- and intermediate-risk groups increased from $32.07 \%$ to $53.69 \%$, yet the percentage in the high-risk group decreased $(\mathrm{P}<0.001)$. The use of radical prostatectomy $(\mathrm{RP})$ and radiation therapy (RT) increased from $48.32 \%$ to $76.46 \%$ and $5.35 \%$ to $16.94 \%$, particularly in high-risk and low-risk groups, respectively, whereas the rates of hormone therapy (HT) and active surveillance and observation (AS\&O) decreased from $32.28 \%$ to $4.27 \%$ and from $16.04 \%$ to $2.33 \%$, respectively $(\mathrm{P}<0.001)$. A similar pattern was observed when patients were stratified by EAU risk group.

Conclusions: The results of this real-world study in the largest regional medical center in Zhejiang Province, China, indicate that the predominant characteristics of PCa patients and trends in initial management are changing rapidly. We found the following: (a) a trend toward a decreased age among newly diagnosed patients; (b) a trend toward lower initial PSA levels; (c) a downward trend in risk group classification; (d) a significant increase in the likelihood of receiving RP, particularly in the high-risk group; (e) an increase in the rate of RP, mostly due to use of the $\mathrm{Da}$ Vinci robotic system; (f) a significant increase in the likelihood of receiving RT, especially in the low-risk group; and (g) a decrease in HT and AS\&O.
\end{abstract}

Key words: prostate cancer (PCa), Patterns of Care Study (PCS), treatment modalities, radical prostatectomy $(\mathrm{RP})$, radiotherapy $(\mathrm{RT})$ 


\section{Introduction}

Prostate cancer (PCa) is the second most common malignancy in men and an important cause of cancer-related morbidity and mortality worldwide [1]. In China, PCa has become the most common malignant tumor of the urinary system and the fastest growing male malignancy [2-5]. However, few reports have examined the characteristics and patterns of PCa treatment in China. Due to interracial differences in the pathological features and clinical manifestations of $\mathrm{PCa}$ and to different perspectives regarding guidelines, treatment modalities vary among physicians. Therefore, an examination of patient characteristics and treatment patterns of $\mathrm{PCa}$ in China is vitally important because these factors may be affected by cultural, racial, ethnic, or social backgrounds [6].

A Patterns of Care Study (PCS) for PCa was first reported by the Department of Radiation Oncology in 1973 [7]. To date, PCSs for PCa have been conducted in several Western countries, which have their own regional or national cancer databases (e.g., the National Cancer Date Base [NCDB], National Program of Registries [NPCR] and Surveillance, Epidemiology, and End Results [SEER] in the USA, the Victorian Admitted Episode Dataset and the Victorian Cancer Registry in Australian, and UK cancer network in the UK). On contrast, China is a developing country that lacks a national cancer database or even provincial databases for studies of PCa treatment modalities.

Our hospital (the First Affiliated Hospital, College of Medicine, Zhejiang University) ranks among China's top 10 hospitals and is the largest medical center in Zhejiang Province. In particular, the urology department of our hospital is ranked the number 1 in Zhejiang both in terms of the number of patients (e.g., Da Vinci surgical urological cases for 2016 and 2017 ranked among the top 3 in China) and treatment technologies (the fourth generation $\mathrm{Da}$ Vinci's robotic system) [8]. Clearly, the medical database of the hospital is quite valuable for PCSs of $\mathrm{PCa}$ in China and represents a real-world situation in China. Therefore, the purpose of this preliminary study was to examine the characteristics of patients and diseases and changes in the patterns of treatment for PCa in the largest regional medical center in China by comparing results for the periods 2010-2011 and 2016-2017 using PCS surveys.

\section{Materials and Methods}

Patients who were newly diagnosed with PCa at First Affiliated Hospital, College of Medicine,
Zhejiang University were enrolled. The following eligibility criteria were used: (a) newly diagnosed for 2010-2011 or 2016-2017; (b) histologically diagnosed with PCa; (c) localized (cT1-2c) and locally advanced (cT3-4 or N+) PCa without distant metastasis; and (d) absence of other malignancies. Detailed information regarding the primary treatment of PCa and patient and disease characteristics were retrospectively collected from the hospital information system (HIS) database by two data collectors. Additionally, initial treatment was defined as management within six months of diagnosis.

A total of 505 and 1,297 patients were included in the 2010-2011 and 2016-2017 surveys, respectively. In this regional medical center PCS survey, patients were categorized into very low-risk, low-risk, intermediaterisk, high-risk, very high-risk, and regional (any T, N1, M0 disease) PCa groups based on National Comprehensive Cancer Network (NCCN) guidelines and into low-risk, intermediate-risk, and high-risk $\mathrm{PCa}$ groups based on European Association of Urology (EAU) guidelines. The patients were also divided into five groups according to their treatment, as follows: (a) a radical prostatectomy (RP) group with or without androgen deprivation therapy (ADT) who underwent postoperative radiotherapy within six months of diagnosis; (b) a radiotherapy (RT) group treated with external beam radiotherapy (EBRT) or ${ }^{125}$ I radioactive seed implantation with ultrasonic guidance with or without ADT; (c) a hormone therapy (HT) group who received only hormone therapy, either with orchiectomy or with administration of drugs to block hormone production or action; and (d) an active surveillance and observation (AS\&O) group who had no record of any therapy within the first 6 months following diagnosis.

The patient and disease characteristics for all patients and the different treatment groups are reported using numbers and frequencies. Differences between proportions were assessed using Pearson's Chi-square test and Fisher's exact test. The Mann-Whitney U-test was used to compare non-parametric continuous data. A P value $<0.05$ was considered significant. Statistical analyses were performed with SPSS, version 22.0 (IBM SPSS, Chicago, IL, USA).

\section{Results}

The patient and disease characteristics obtained from the PCS surveys for the 2010-2011 and 2016-2017 periods are shown in Table 1. Compared with the ages of the patients diagnosed in 2010-2011, there was a 
distinct trend toward a lower age in the 2016-2017 PCS $(\mathrm{P}<0.001)$. In both PCS surveys, the proportion of patients with T2 was greater than $50 \%$ and reached $74.39 \%$ in the 2016-2017 PCS. N-stage distribution in the patient population did not differ significantly between the 2010-2011 and 2016-2017 surveys, with nearly $90 \%$ having N0-stage disease. There was a marked decrease in the level of initial prostate-specific antigen (PSA) in the 2016-2017 PCS survey compared with that in the 2010-2011 PCS survey. From 2010-2011, approximately $50.69 \%$ of patients had an initial PSA level $\geq 20 \mathrm{ng} / \mathrm{ml}$. However, $66.67 \%$ had an initial PSA level of 4-19.99 $\mathrm{ng} / \mathrm{ml}$ in the 2016-2017 PCS survey $(\mathrm{P}<0.001)$. Based on NCCN criteria, the percentages of patients in the low-risk and intermediate-risk groups increased from $33.06 \%$ to $54.78 \%$, whereas the percentages of patients in the high-risk, very high-risk, and regional (N1) groups decreased significantly $(\mathrm{P}<0.001)$. Based on EAU criteria, the percentages of patients in the low-risk and intermediate-risk groups increased from $32.07 \%$ to $53.69 \%$, and the percentage of patients in the high-risk group decreased $(\mathrm{P}<0.001)$.

The primary treatment and changing trends for all PCa patients (localized and locally advanced) and the management trends stratified by risk group over the 6-year period are presented in Figure 1. Compared with the 2010-2011 PCS survey, the rate of curative treatment (RP and RT) increased in the 2016-2017 PCS survey (Figure 1A). Notably, the rate of RP increased dramatically from $48.32 \%$ to $76.46 \%$. Interestingly, a similar pattern was observed for patients with high-risk disease, with RP increasing from $41.40 \%$ to $72.65 \%$ (Figure 1D). An increase in the proportion of RT was observed in the low-risk group, from $4.65 \%$ to $17.07 \%$ (Figure 1B). Conversely, the percentages of patients undergoing $\mathrm{HT}$ and $\mathrm{AS} \& \mathrm{O}$ decreased markedly from $32.28 \%$ to $4.27 \%$ and from $16.04 \%$ to $2.33 \%$, respectively (Figure 1A). The same trend was observed in the intermediate-risk group (Figure 1B).

The proportions of patients in the RP group who received different surgical approaches are shown in Figure 2. Patients at our medical institution received either open retropubic, laparoscopic, or Da Vinci robotic RP. The percentage of patients who received $\mathrm{Da}$ Vinci robotic RP increased significantly, overtaking open retropubic RP as the most common surgical approach because only open retropubic or laparoscopic RP was performed during the 2010-2011 period. From 2016 to 2017, the percentage of patients undergoing open retropubic RP remained below 30\%. Remarkably, once-popular laparoscopic surgery also suffered a decline. Further details are provided in Figure 2.
Table 1. Characteristics of PCa patients and disease in 2010-2011 versus 2016-2017.

\begin{tabular}{|c|c|c|c|}
\hline Characteristics & $2010-2011(n=505)$ & $2016-2017(n=1287)$ & $\mathbf{P}$ \\
\hline Age(years) & & & $<0.001$ \\
\hline mean & 70 & 68 & $<0.001$ \\
\hline $30-39$ & $0(0.00 \%)$ & $1(0.08 \%)$ & \\
\hline $40-49$ & $5(0.99 \%)$ & $6(0.47 \%)$ & \\
\hline $50-59$ & $42(8.32 \%)$ & $124(9.63 \%)$ & \\
\hline $60-69$ & $170(33.66 \%)$ & $586(45.53 \%)$ & \\
\hline $70-79$ & $230(45.54 \%)$ & $486(37.76 \%)$ & \\
\hline $80-89$ & $56(11.09 \%)$ & $84(6.53 \%)$ & \\
\hline$\geq 90$ & $2(0.42 \%)$ & $0(0.00 \%)$ & \\
\hline cT stage & & & $<0.001$ \\
\hline $\mathrm{T} 1$ & $8(2.51 \%)$ & $51(6.60 \%)$ & \\
\hline $\mathrm{T} 2$ & $171(53.61 \%)$ & $575(74.39 \%)$ & \\
\hline $\mathrm{T} 3$ & $112(35.11 \%)$ & $131(16.95 \%)$ & \\
\hline $\mathrm{T} 4$ & $28(8.78 \%)$ & $16(2.07 \%)$ & \\
\hline cN stage & & & 0.806 \\
\hline No & $292(91.54 \%)$ & $704(91.07 \%)$ & \\
\hline N1 & $27(8.46 \%)$ & $69(8.93 \%)$ & \\
\hline initial PSA & & & $<0.001$ \\
\hline$<4$ & $32(6.34 \%)$ & $19(1.48 \%)$ & \\
\hline $4-10.0$ & $96(19.01 \%)$ & $449(34.89 \%)$ & \\
\hline $10.01-19.99$ & $121(23.96 \%)$ & $409(31.78 \%)$ & \\
\hline $20-49.99$ & $104(20.59 \%)$ & $258(20.05 \%)$ & \\
\hline$\geq 50$ & $152(30.10 \%)$ & $152(11.81 \%)$ & \\
\hline Gleason score & & & $<0.001$ \\
\hline$\leq 6$ & $111(24.45 \%)$ & $338(27.35 \%)$ & \\
\hline $3+4$ & $84(18.50 \%)$ & $287(23.22 \%)$ & \\
\hline $4+3$ & $72(15.86 \%)$ & $242(19.58 \%)$ & \\
\hline 8-9 no primary 5 & $148(32.60 \%)$ & $312(25.24 \%)$ & \\
\hline Any primary 5 & $39(8.59 \%)$ & $57(4.61 \%)$ & \\
\hline Risk group/NCCN & & & $<0.001$ \\
\hline very low risk & $0(0.00 \%)$ & $0(0.00 \%)$ & \\
\hline low risk & $43(8.51 \%)$ & $123(9.56 \%)$ & \\
\hline intermediate risk & $124(24.55 \%)$ & $582(45.22 \%)$ & \\
\hline high risk & $190(37.62 \%)$ & $376(29.22 \%)$ & \\
\hline very high risk & $120(23.76 \%)$ & $140(10.88 \%)$ & \\
\hline Regional/N1 & $28(5.54 \%)$ & $66(5.13 \%)$ & \\
\hline Risk group/EAU & & & $<0.001$ \\
\hline low risk & $43(8.51 \%)$ & $123(9.56 \%)$ & \\
\hline intermediate risk & $119(23.56 \%)$ & $568(44.13 \%)$ & \\
\hline high risk & $343(67.92 \%)$ & $596(46.31 \%)$ & \\
\hline
\end{tabular}

consistent due to absence of pelvic MRI data.

\section{Discussion}

The present study indicates that the patterns of care for localized and locally advanced PCa without distant metastasis changed rapidly in the largest regional medical center in Zhejiang Province, China, over the study period.

There was a trend toward a lower age at diagnosis and a lower initial PSA level in the 2016-2017 PCS survey compared with the 2010-2011 PCS survey, as well as a downward shift in T stage. Such a tendency has also been observed in Western countries [9]. This phenomenon may be due to improvements in healthcare, patient awareness of their own health, and increasingly early detection (e.g., PSA screening, digital rectal examination). Our study revealed that $29.22 \%$ of patients were classified with high-risk disease in the 2016-2017 PCS survey; similarly, in 2016, one-third of the patients diagnosed with localized PCa were classified as 'high risk' in the USA based on NCCN criteria [10]. 
A

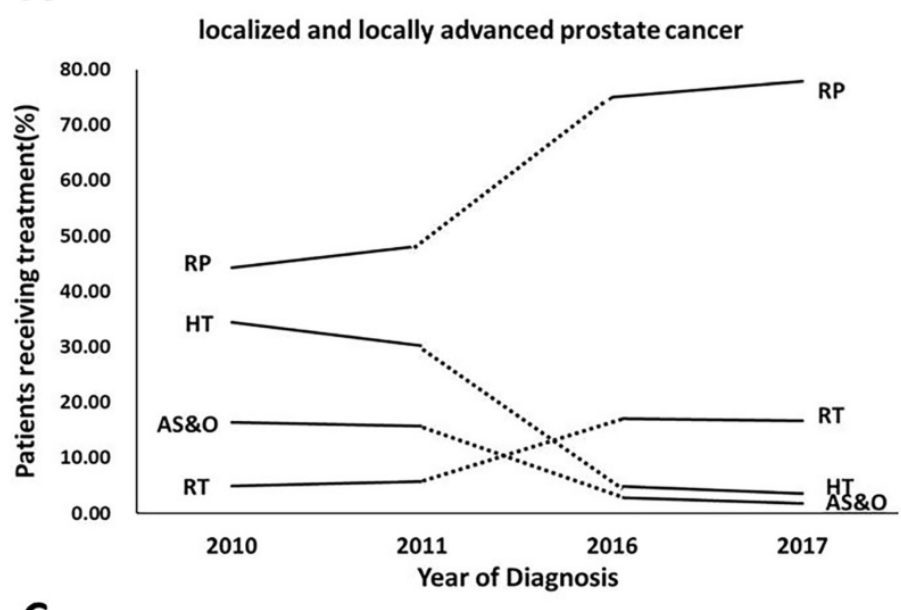

C

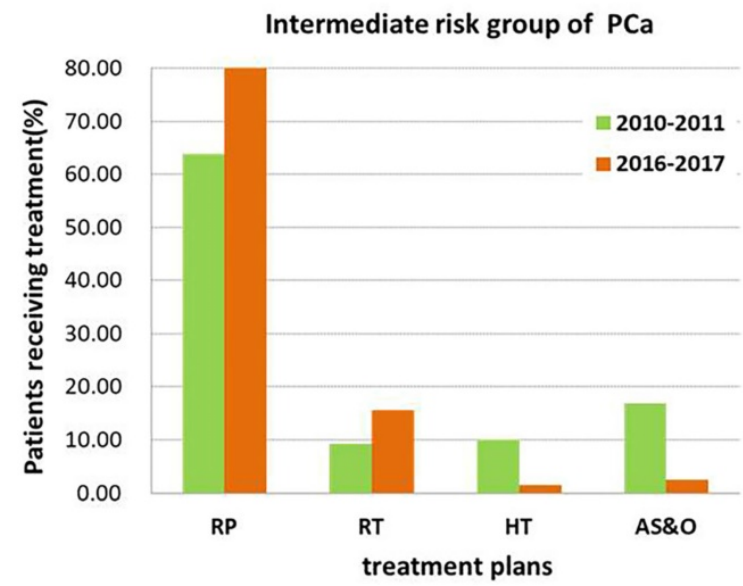

B

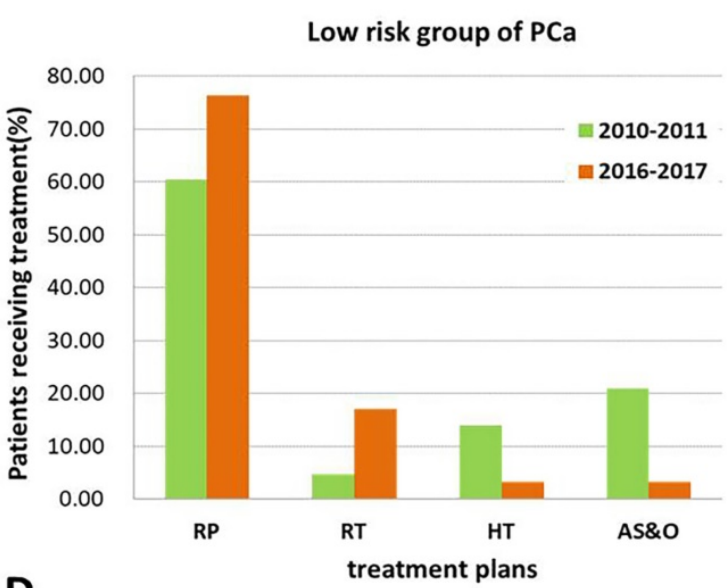

D

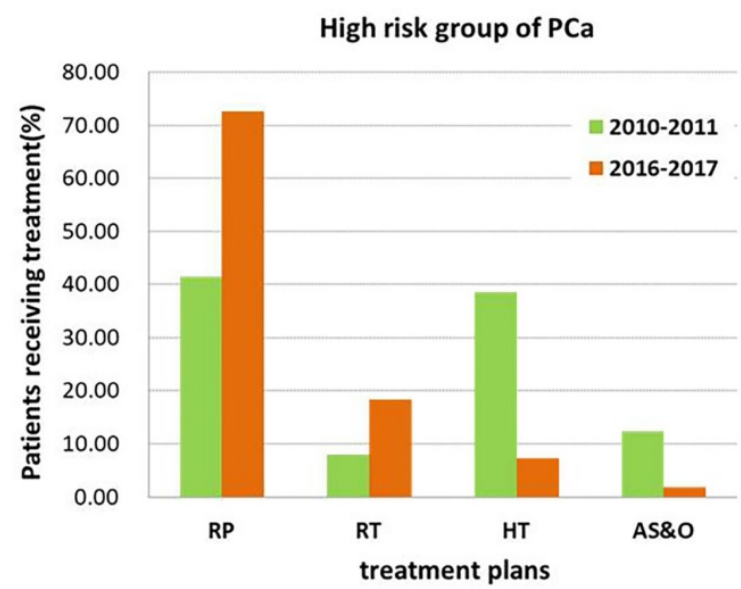

Figure 1: The rates of primary treatment (RP, RT, HT, and AS\&O) for all localized and locally advanced PCa patients for (A) the low-risk group of PCa patients, (B) the intermediate-risk group of PCa patients, (C) and the high-risk group of PCa patients (D) from 2010 to 2011 (green) and from 2016 to 2017 (orange).

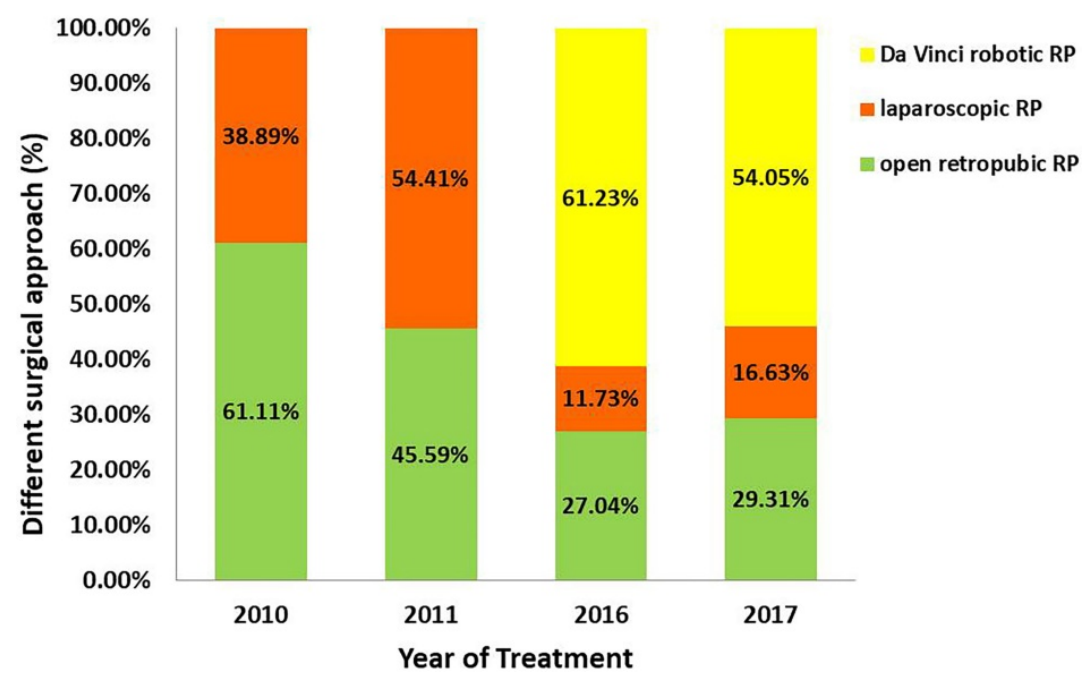

Figure 2: The percentage of patients receiving different surgical approach in the RP group: open retropubic (green), laparoscopic (orange), or Da Vinci robotic RP (yellow).

According to current NCCN Clinical Practice Guidelines, observation, active surveillance, radiation, and radical prostatectomy are all reasonable treatment options for localized and locally advanced $\mathrm{PCa}$, and these recommendations have not changed significantly in recent years [11]. However, 
there is no consensus to date among physicians in China regarding treatment options for PCa. Discrepancies in physician opinions, particularly across subspecialties, may have contributed to the diverse treatments used for PCa.

In our study, the percentage of patients who underwent definitive treatment (e.g., RP or RT) increased, whereas the percentage of patients who underwent conservative treatment (e.g., HT or AS\&O) decreased. This finding may be due to the improved awareness of patients and to advanced research into diverse treatment plans. In addition, RP was the predominant treatment in both surveys, though the RT rate at our institution remained considerably low. However, a similar study conducted in 2010 revealed that the RT rate exceeded the RP rate $(42 \%$ versus $39 \%)$ [11].

In our study, the proportion of PCa patients who received RP increased to nearly 75\% in the 2016-2017 survey, a rate that was maintained at over $50 \%$ in the USA [6], and in our study, this tendency was particularly observed in patients with high-risk disease. Several factors may have contributed to the increase in RP rate. For example, it has been reported that an improved cancer-specific survival benefit is associated with RP compared with RT [12, 13]. Additionally, a survival benefit has also been found for men at high risk for PCa [14-17]. Another reason may be that the majority of Chinese PCa patients are initially diagnosed by urologists; therefore, the dominant use of surgery as a curative therapy may be attributable to clinician factors [18]. Moreover, the introduction of Da Vinci robotic surgery in 2014 increased the number of surgeries in PCa patients and provided another surgical option for the management of patients with $\mathrm{PCa}$, especially for those with high-risk disease. According to Intuitive Surgical records, more than 2,400 surgical cases were completed at First Affiliated Hospital of Zhejiang University using the Da Vinci system. Indeed, as use increased, our center set a world record, 836 cases, in 2015 for single-unit use, which was broken when our center reached 888 cases in 2016 [8]. Furthermore, robot-assisted RP is safe in men $>75$ years of age and has low complication rates, good oncologic and continence outcomes, and reasonable potency outcomes [19]. Therefore, RP is an adequate treatment option in carefully selected elderly men with a good health status [20-22].

Only $16.94 \%$ of PCa patients received RT using either an external beam or ${ }^{125} \mathrm{I}$ radioactive seed implantation in our 2016-2017 PCS survey, which was in stark contrast to the proportion of almost $35 \%$ in the USA [6]. Although the rate of RT is still very low in China, this method is gradually becoming accepted as an alternative option for curative treatment of PCa (5.35\% for $2010-2011$ versus $16.94 \%$ for $2016-2017$ ), especially for patients with low-risk disease. This change may be occurring because patients have become more informed about RT as an available curative treatment option and because the general public has become more aware of the advantages; additionally, Chinese physicians may be following guidelines more closely. Nevertheless, considering that more than $30 \%$ of non-metastatic PCa patients undergo RT in the USA [23, 24], the prevalence of RT for PCa in our study was very low. A possible reason for the low prevalence of RT may be a lack of considerable population-based studies revealing the safety and reliability of RT. Another reason may be inadequate dissemination of information on the application and advantages of RT.

The proportion of PCa patients who received HT in our study decreased dramatically to $4.27 \%$ in the 2016-2017 period, which was consistent with the proportion in the USA (approximately 3\%) [6], particularly in patients with high-risk disease (a decrease from $38.48 \%$ to $7.21 \%$ ). Reduced use of primary ADT is in line with current practice guidelines, and this finding is encouraging given evidence demonstrating the lack of survival benefit associated with primary ADT, especially among high-risk patients [25, 26]. However, patients who underwent HT as their primary strategy may have undergone a secondary treatment 6 months after diagnosis due to their concern of under-treatment, particularly those who had an adequate understanding of PCa and acceptably recommended treatment options. These patients were still classified in the HT group, which might explain the high rate of HT as the initial treatment in the 2010-2011 PCS survey.

The proportion of PCa patients who received AS\&O in our study decreased from $16.04 \%$ to $2.33 \%$; this proportion was approximately $10 \%$ in the USA. Many studies have attempted to explain the reasons for the decreased utilization of AS\&O, an approach that is often preferred to avoid overtreatment in elderly men with PCa with a limited life expectancy and multiple comorbidities [27]. Although there is evidence to suggest that men with indolent cancers can be safely observed, in the study by Godtman RA et al., this option was deemed safe only for men with very low-risk PCa [28]. Furthermore, in patients with low- and intermediate-risk PCa, this strategy carries a risk of missing the opportunity for a cure. Another study found that patients who were $\geq 75$ years old had a higher rate of being upgraded to a higher disease grade based on RP specimens [29]. Therefore, it remains controversial whether AS\&O can be safely 
recommended for all elderly patients.

Our study is not without its limitations. One limitation was that the analysis was not based on the entire national PCa population due to the lack of a national cancer database in China. Another limitation was that the study was a single-institution, retrospective review without a control group, and it is unknown whether First Affiliated Hospital, College of Medicine, Zhejiang University is a suitable source of data from which to examine patterns of care for PCa. Despite these limitations, to the best of our knowledge, a complete assessment of primary treatments in China for PCa has not been reported, and our study presents the first evaluation of changing trends in initial treatment strategies for PCa. Thus, the results will provide useful information for evaluating recent dominant trends in treatment plans for PCa. The establishment of a national database study will provide better insight for defining standard guidelines for PCa treatment in China.

\section{Conclusion}

This PCS was performed in the largest regional medical center in Zhejiang Province to identify recent predominant trends in treatment plans for local and locally advanced PCa in China and revealed the following: (a) a trend toward a lower age in newly diagnosed patients; (b) a trend toward lower initial PSA values; (c) a trend toward a downward shift in T stage and risk groups; (d) a significant increase in the likelihood of receiving $\mathrm{RP}$, especially in the high-risk group; (e) an increase in the rate of RP, mostly due to use of the Da Vinci robotic system; (f) a significant increase in the likelihood of receiving RT, especially in the low-risk group; and (g) a decrease in the use of HT and AS\&O.

\section{Abbreviations}

PCa: prostate cancer;

PCS: Patterns of Care Study;

HIS: hospital information system;

PSA: prostate-specific antigen;

RP: radical prostatectomy;

RT: radical radiotherapy;

EBRT: external beam radiotherapy;

ADT: androgen deprivation therapy;

AS\&O: active surveillance and observation;

NCCN: National Comprehensive Cancer

Network;

EAU: European Association of Urology.

\section{Acknowledgements}

We thank the nurses at the department of radiation oncology ( $\mathrm{Yu}$ LI, Wei WEI, Qun WU, Huaqin ZHU, Xiaolian ZHANG, and Fang TONG) for their help with data collection.

\section{Competing Interests}

The authors have declared that no competing interest exists.

\section{References}

1. Siegel RL, Miller KD, Jemal A. Cancer statistics, 2017. CA Cancer J Clin. 2017; 67(1): 7-30.

2. Zheng $\mathrm{R}$, Zeng $\mathrm{H}$, Zhang $\mathrm{S}$, et al. National estimates of cancer prevalence in China, 2011. Cancer Lett. 2016; 370(1): 33-8.

3. Chen W, Zheng R, Zeng H, et al. Annual report on status of cancer in China, 2011. Chin J Cancer Res. 2015; 27(1): 2-12.

4. Na R, Ye D, Qi J, et al. Prostate health index significantly reduced unnecessary prostate biopsies in patients with PSA $2-10 \mathrm{ng} / \mathrm{mL}$ and PSA $>10 \mathrm{ng} / \mathrm{mL}$ : Results from a Multicenter Study in China. Prostate. 2017; 77(11): 1221-9.

5. Chen W, Zheng R, Baade PD, et al. Cancer statistics in China, 2015. CA Cancer J Clin. 2016; 66(2): 115-32.

6. Gray PJ, Lin CC, Cooperberg MR, et al. Temporal Trends and the Impact of Race, Insurance, and Socioeconomic Status in the Management of Localized Prostate Cancer. Eur Urol. 2017; 71(5): 729-37.

7. Chuba PJ, Moughan J, Forman JD, et al. The 1989 patterns of care study for prostate cancer: five-year outcomes. Int J Radiat Oncol Biol Phys. 2001; 50(2): 325-34.

8. He Z, Zeng L, Zhang C, et al. Initial experience of Da Vinci robotic thoracic surgery at the First Affiliated Hospital of Zhejiang University. J Vis Surg. 2017; 3: 153 .

9. Nakamura K, Ogawa K, Yamamoto T, et al. Trends in the practice of radiotherapy for localized prostate cancer in Japan: a preliminary patterns of care study report. Jpn J Clin Oncol. 2003; 33(10): 527-32.

10. Mohler JL, Armstrong AJ, Bahnson RR, et al. Prostate Cancer, Version 1.2016. J Natl Compr Canc Netw. 2016; 14(1): 19-30.

11. Schymura MJ, Kahn AR, German RR, et al. Factors associated with initial treatment and survival for clinically localized prostate cancer: results from the CDC-NPCR Patterns of Care Study (PoC1). BMC Cancer. 2010; 10: 152.

12. Barry MJ, Albertsen PC, Bagshaw MA, et al. Outcomes for men with clinically nonmetastatic prostate carcinoma managed with radical prostactectomy, external beam radiotherapy, or expectant management: a retrospective analysis. Cancer. 2001; 91(12): 2302-14.

13. Lu-Yao GL, Yao SL. Population-based study of long-term survival in patients with clinically localised prostate cancer. Lancet. 1997; 349(9056): 906-10.

14. Wallis CJD, Saskin R, Choo R, et al. Surgery Versus Radiotherapy for Clinically-localized Prostate Cancer: A Systematic Review and Meta-analysis. Eur Urol. 2016; 70(1): 21-30.

15. Cooperberg MR, Vickers AJ, Broering JM, et al. Comparative risk-adjusted mortality outcomes after primary surgery, radiotherapy, or androgen-deprivation therapy for localized prostate cancer. Cancer. 2010; 116(22): 5226-34

16. Petrelli F, Vavassori I, Coinu A, et al. Radical prostatectomy or radiotherapy in high-risk prostate cancer: a systematic review and metaanalysis. Clin Genitourin Cancer. 2014; 12(4): 215-24.

17. Zelefsky MJ, Eastham JA, Cronin AM, et al. Metastasis after radical prostatectomy or external beam radiotherapy for patients with clinically localized prostate cancer: a comparison of clinical cohorts adjusted for case mix. J Clin Oncol. 2010; 28(9): 1508-13.

18. Park J, Suh B, Shin DW, et al. Changing Patterns of Primary Treatment in Korean Men with Prostate Cancer Over 10 Years: A Nationwide Population Based Study. Cancer Res Treat. 2016; 48(3): 899-906.

19. Labanaris AP, Witt JH, Zugor V. Robotic-assisted radical prostatectomy in men $\geq 75$ years of age. Surgical, oncological and functional outcomes. Anticancer Res. 2012; 32(5): 2085-9.

20. Mandel P, Kriegmair MC, Kamphake JK, et al. Tumor Characteristics and Oncologic Outcome after Radical Prostatectomy in Men 75 Years Old or Older. J Urol. 2016; 196(1): 89-94.

21. Kunz I, Musch M, Roggenbuck U, et al. Tumour characteristics, oncological and functional outcomes in patients aged $\geq 70$ years undergoing radical prostatectomy. BJU Int. 2013; 111(3 Pt B): E24-9.

22. Xylinas E, Ploussard G, Paul A, et al. [Laparoscopic radical prostatectomy in the elderly (>75 years old): oncological and functional results]. Prog Urol. 2010; 20(2): 116-20.

23. Aneja S, Smith BD, Gross $\mathrm{CP}$, et al. Geographic analysis of the radiation oncology workforce. Int J Radiat Oncol Biol Phys. 2012; 82(5): 1723-9.

24. Mahmood U, Levy LB, Nguyen PL, et al. Current clinical presentation and treatment of localized prostate cancer in the United States. J Urol. 2014; 192(6): 1650-6.

25. Mason MD, Parulekar WR, Sydes MR, et al. Final Report of the Intergroup Randomized Study of Combined Androgen-Deprivation Therapy Plus Radiotherapy Versus Androgen-Deprivation Therapy Alone in Locally Advanced Prostate Cancer. J Clin Oncol. 2015; 33(19): 2143-50. 
26. Widmark A, Klepp O, Solberg A, et al. Endocrine treatment, with or without radiotherapy, in locally advanced prostate cancer (SPCG-7/SFUO-3): an open randomised phase III trial. Lancet. 2009; 373(9660): 301-8.

27. Mottet N, Bellmunt J, Bolla M, et al. EAU-ESTRO-SIOG Guidelines on Prostate Cancer. Part 1: Screening, Diagnosis, and Local Treatment with Curative Intent. Eur Urol. 2017; 71(4): 618-629.

28. Godtman RA, Holmberg E, Khatami A, et al. Long-term Results of Active Surveillance in the Göteborg Randomized, Population-based Prostate Cancer Screening Trial. Eur Urol. 2016; 70(5): 760-766.

29. Herlemann A, Buchner A, Kretschmer A, et al. Postoperative upgrading of prostate cancer in men $\geq 75$ years: a propensity score-matched analysis. World J Urol. 2017; 35(10): 1517-1524 